\begin{tabular}{|c|c|c|}
\hline 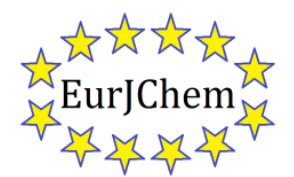 & $\begin{array}{c}\text { European Journal of Chemistry } \\
\text { Journal homepage: } \text { www.eurjchem.com }\end{array}$ & 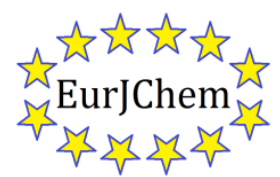 \\
\hline
\end{tabular}

\title{
On shapes, molecules and models: An insight into chemical methodology
}

\author{
Mario Pagliaro \\ Istituto per lo Studio dei Materiali Nanostrutturati, CNR, via U. La Malfa 153, Palermo, IT-90146, Italy
}

Corresponding author at: Istituto per lo Studio dei Materiali Nanostrutturati, CNR, via U. La Malfa 153, Palermo, IT-90146, Italy. Tel.: +39.091.6809370; fax: +39.091.6809247. E-mail address: mario.pagliaro@cnr.it (M. Pagliaro).

\section{ARTICLE INFORMATION}

Received: 04 June 2010

Accepted: 03 September 2010

Online: 31 December 2010

\section{KEYWORDS}

Chemical methodology

Chemical education

Reductionism

Quantum chemistry

Models in chemistry

\begin{abstract}
Insight into chemistry conceptual foundations shows that a powerful, autonomous chemical methodology exists which is based on visualization and association of chemical "building blocks". Such methodology has been expanded by the use of incommensurable theories resulting from the interplay of quantum mechanics and heuristic chemical concepts, resulting in models of great utility as predictive tools in guiding the creation of new and useful substances. Understanding such historical development has real benefits to chemistry practitioners, be they researchers, analysts or chemical educators.
\end{abstract}

\section{Introduction}

Asked why two electrically negative particles called electrons should actually bind two atoms together, our student remained puzzled. "Both negative? I never thought about that". Probably, this student is not alone. After all, chemists are experimental scientists supposed to spend most of their time mixing smelly stuff in the laboratory without devoting much attention to these conceptual details. And yet, is this true? What is the conceptual difference between what the layman thinks the practice of chemistry is, and what it actually is?

Should we tell the student that that electrons are no more than "knots in the fabric of spacetime" and demonstrate her/him how this idea enables us to understand many of their properties, including their spin? [1] Paul Dirac, for instance, as early as in 1929 had little doubt claiming that: [2]

"The underlying laws necessary for the mathematical theory of a large part of physics and the whole of chemistry are thus completely known, and the difficulty is only that the exact application of these laws leads to equations much too complicated to be soluble. It therefore becomes desirable that approximate practical methods of applying quantum mechanics should be developed, which can lead to an explanation of the main features of complex atomic systems without too much computation".

According to this view chemistry will be considered as a branch of physics; and dealt with accordingly also from the teaching viewpoint.

Most of today's chemistry textbooks start with a chapter featuring "electron clouds" claimed to be fully equivalent with "orbitals" [3]. The chemical bond is there described more or less as a spring connecting two fixed, spherical atoms constituting part of the molecule. And even if such molecules do not seem much different from what Ampere in 1814 had -astonishingly -- suggested "simple polyhedra in which the atoms occupy the corners" with the polyhedron representing "the representative form of the particle", [4] still in the early 1990s the author of a respected physical chemistry textbook also left little doubt: "Matter is made of atoms because we can see them" [5].

The approach to structural chemistry, however, remained heuristic and often cumbersome. For instance, do electrons in most organic chemistry molecules organize so as to be eight in the "outer electron shell"? Then we may invoke an "octet rule"; a rule holding true in few cases if, however, in 1986 the authors of another recognised chemistry textbook could write:

"You may be wondering if the structures that you learned previously (which followed the octet rule) are wrong. They are not wrong, but are not the best structures that can be given for these molecules" [6].

Indeed, only ten years later theoretical chemists discussing the leading "resonance" structures of simple inorganic anions such as $\mathrm{SO}_{4}^{2-}, \mathrm{ClO}_{4}^{-}, \mathrm{PO}_{4}{ }^{3-}$ cited in freshman chemistry textbooks, argued that such structures "are not the most accurate to represent the molecule" and that the most accurate structures are "the original Lewis structures that generally abide by the octet rule" [7].

Pimentel was amongst the first to react to this confusing exposition of chemistry theory lamenting that students were being accosted with " $\pi$-bonds, bridge-bonds, one-electron bonds" [8]; and by the end of 1960s he proposed radical changes in the way chemistry was being taught. Similarly, Cotton and Wilkinson published their 1962 landmark textbook of inorganic chemistry aiming to make the academic community "conscious of the need to teach molecular orbitals and to encourage the use of group theory" [9].

The underlying idea, however, was still that of Dirac, i.e., the theory of chemistry was well understood in terms of quantum mechanics and that chemical central issues such as reactivity, kinetics and chirality were just matter of calculation power (and thus of time).

Still, how far all this could be from the practice of the chemical enterprise apparently did not disturb contemporary chemists very much. A science whose conceptual model had 
been weakened by the 1930s revolution of quantum mechanics remained confusing in its foundations even to its best practitioners. For example, this author still remembers his organic chemistry professor who cautioned his students in the early 1990s to "forget molecular orbitals. If you think you can do chemistry with molecular orbitals, you will never achieve anything" [10]. Today, as we witness to a worldwide decline in the enrolment of students in chemistry courses in all major scientifically advanced countries (with a trend similar in the UK, Europe, and the US) [11] we argue that restoring the very concept that chemistry is an autonomous science with both an intrinsic logic and methodology -- the aim of this concept article -- is of fundamental importance to reverse this situation and inspire new enthusiasm among chemistry practitioners, who historically deal with acute societal problems, and yet suffer from a poor public image [12].

\section{Chemistry on a logical basis}

The pillars of chemistry are two, namely analysis and synthesis. Chemical analysis enables to find out which elements -- operationally defined by Lavoisier as "the furthest stage to which analysis can reach" -- compose a substance; chemical synthesis allows chemists to create new substances or to devise new routes to compounds created by Nature. Of course, the complexity of the substances that chemists can analyze and synthesize, as well as the ease of either processes, has increased enormously over the past 80 years thanks to the progress made both in theory and in the available experimental tools. A wealth of specialized reagents to carry out specific transformations; chromatography to isolate and analyze substances of interest; spectroscopy and other physical methods to rapidly elucidate the structures of unknown compounds.

In the late 1920s chemists developed what Corey calls "one of the great scientific revolutions of the 20th century", i.e., a theory of chemical reactions based on rearrangement of electron pairs as bonds are made and broken, opening the route to design synthetic routes and ultimately "putting organic synthesis on a logical basis" [13]. Getting to reactivity, for instance, chemists started to explain the combinatorial rules (reactivity paths) for which under certain precise experimental conditions (which, in chemistry, are crucially important), pure substances behave according to these rules, in terms of electron chemical bonds scission and formation. In this manner the old rules based on repeated observation, abstraction and generalization started to find a logical explanation. For instance, the reaction between an amine and a ketone generally occurs with elimination of water so that a reaction scheme can be generalized as in Equation 1:

$$
\mathrm{R}-\mathrm{NH}_{2}+\mathrm{R}^{\prime} \mathrm{R}^{\prime \prime}-\mathrm{CO} \rightarrow \mathrm{R}-\mathrm{N}=\mathrm{C}-\mathrm{R}^{\prime} \mathrm{R}^{\prime \prime}+\mathrm{H}_{2} \mathrm{O}
$$

A mechanistic viewpoint which makes use of ideas such as molecular structure, valence and electrons and so on is then applied to justify observations. But certainly, no physicist would find this intellectual process anyhow close to what he/she actually does to interpret the results of physical experiments. For example, it is sufficient to analyse a recent theoretical study [14] describing chemical reactivity by quantum chemical methods, to recognize that most of the heuristic chemical concepts -- electronegativity, electron affinity, electron donor and acceptor, chemical "hardness" and "softness", valence, etc. --, are still employed by today's theoretical chemists.

Yet, chemists using their unique methodology have been able to synthesize a cornucopia of incredibly useful substances. This is what gives chemists their power and scientific importance and not what Ogilvie calls "the self-imposed tyranny" [15] of the Schrödinger equation (Equation 2, for the $N$-electron wavefunction $\Psi$ ).

$$
\begin{aligned}
& \mathrm{H} \Psi(N)=E \Psi(N) \\
& \psi_{j}=\sum_{i=1}^{n} c_{i j} \chi_{i}
\end{aligned}
$$

An equation that is generally solved under the assumption of fixed nuclei of the Born-Oppenheimer approximation; and of the self-consistent field (SCF) theory that each electron moves in a spherically symmetrical field so that the equation can be solved for each particle separately, then summed together. In other words, in this theory, it is assumed that the molecular orbital wavefunction $\Psi_{f}$ may be written as a simple weighted sum of the $N$ constituent one-electron wavefunctions called atomic orbitals $\chi_{i}$ (Equation 3) [16].

Now, pretending that empirical rules used by chemists to interpret repeated observations (such as the lack of reactivity of noble gas elements with heuristic concepts such as the octet rule) are of fundamental nature, may easily implicate a limitation to the progress of chemistry; as the case of the delayed discovery of xenon compounds ("forbidden" by the octet rule) typically demonstrates. Indeed, we had to wait until 1962 when Bartlett recognized that since the ionization potential of xenon is almost identical with that of $\mathrm{O}_{2}$, its replacement with $\mathrm{Xe}$ in the reaction with $\mathrm{PtF}_{6}$ would give the compound $\mathrm{XePtF}_{6}$, as actually shown to be the case [17].

Remarkably, the discovery of electron deficient molecules had already put the octet rule in question in the mid 1950s, mostly by R.E. Rundle who pioneered the theoretical elucidation of electron deficient compounds in terms of molecular orbitals. In particular, he explained by this approach the three-center four-electron bond that held the $\mathrm{I}_{3}$ ion together, which he had encountered in his X-ray analyses of starch-iodine complexes [18]. On the bases of this reasoning, he then predicted the existence of linear noble-gas halogen compounds. Thus the quantum mechanics-based reasoning proved superior to the older non-quantum mechanical empirical rules.

A closer look to how the chemical ingenuity is out in action adds insight into chemical methodology and throws light on the concepts of chemical structure and chemical bond.

\section{Visualization and association: The methodology of chemistry}

From a chemist's viewpoint, the concept of molecular structure is a functional model that is essential for predicting the outcome of chemical reactions not yet carried out. What chemists really do is to create mental images of the substances they wish to create - and then manipulate these forms (shapes) in a rational manner to verify if they could fit to afford the desired substance. Kekulé's speech at a dinner commemorating his "discovery" of benzene's structure (Figure 1) renders this process vividly: [19]

"The atoms were gamboling before my eyes... My mental eye, rendered more acute by repeated vision of this kind, could not distinguish larger structures, of manifold conformation; long rows, sometimes more closely fitted together; all twining and twisting in snakelike motion. But look! What was that? One of the snakes had seized hold of its own tail, and the form whirled mockingly before my eyes."

Therefore, the molecular structure is not, in and of itself, a manifestation of the notion that chemistry is subordinate to physics, but rather a powerful model that chemists can actually use. 


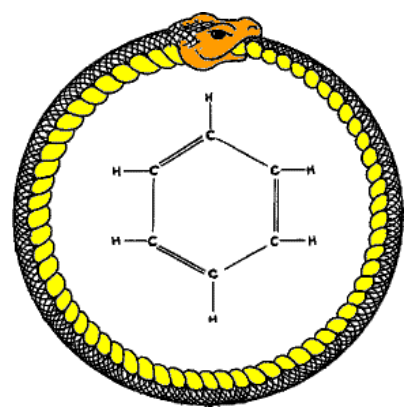

Figure 1. The structure of benzene revealed as a mental image of a snake to Kekulé. (Reproduced from Ref. [19], with permission).

Showing how fertile this visualization approach continues to be today, let us for example take symmetry, a concept of fundamental importance in chemistry. Its chemical usefulness had traditionally been limited to application of Group theory to crystal classification and solution of the Schrödinger equation. Then, in the early 1990s the concept of continuous symmetry measure (CSM, a number, on a scale from zero to 100, that allows to evaluate the degree of symmetry of an object) was introduced [20] using exactly the visualization process typical of chemists: [21]

"Consider the following series of substituted 2-butanes (Figure 2): 2-fluorobutane and 2-iodobutane are of course chiral, but so is 2-deuteriobutane, which is only marginally different from the parent achiral butane... intuition... probably dictates correctly that since the 2-deuterio derivative is actually not that different from the achiral n-butane, its "degree of chirality" is quite small.

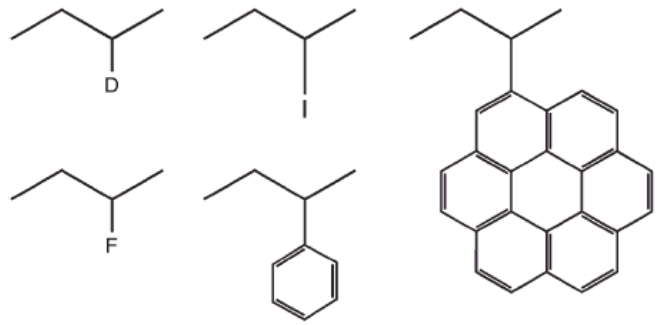

Figure 2. Series of substituted 2-butanes. (Reproduced from Ref. [21], with permission).

"Likewise... iodobutane is perhaps "more chiral" than fluorobutane, because the iodine atom is much larger than the fluorine atom, and therefore disturbs more the achirality of butane. Increasing even more the 2-substituent, one can perhaps say that 2-phenylbutane is "highly chiral", but if the very large coronene is used as a substituent, then the chirality of 2coronenobutane is not so pronounced, because the butyl substituent on the very large polycyclic molecule is just a small disturbance to its achirality."

Getting back to the practice of chemistry, we may recognize that what chemists actually do practicing their science is to mentally play with these representations before entering the lab. Said another way, the chemist's mind proceeds by selfcreating an image (Figure 3) of how atoms, molecules and other matter building blocks will actually behave, and then tries to mentally "push" them on the desired route.

In this sense, learning the practice of chemistry is analogous to learning a language. In each language, there are rules to combine the elementary units (words) which in their turn represent objects and ideas. And the result of the combination is a meaningful language that enables people to communicate. In the chemical practice, the units are the chemist's building blocks and the outcome is a new substance whose structure and functions are to be discovered. Chemistry in its own constitutes a language; and it is remarkable to notice how this concept is commonly shared within the chemical community: [22]
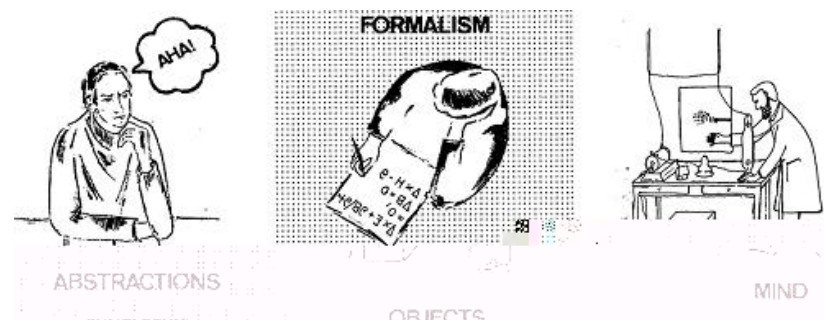

Figure 3. Chemists create mental images of the behaviour of matter. And then go the lab to create their objects: the myriad substances benefiting society at large. (Reproduced from Ref. 39b, with permission).

"A chemical formula is like a word. It purports to identify, to single out the chemical species it stands for... but it remains a long, long way from the molecular scale to the macroscopic world of the senses. We still have to represent molecules...And we tend to represent atoms as if they were normal objects in our everyday experience". In a modern plural view, thus, the molecular structure becomes a model for the substance, namely a representation of it or, better said, " $a$ symbolic transformation of reality, at once a model and a theoretical construct" [22].

\section{Useful, incommensurable models}

Following the introduction of advanced spectroscopic and analytical instrumentation in the late 1950s, chemists became able to obtain the structure of the substances created in their experiments at an unprecedented rate, and thus check the match between the visualization process mentioned above and results of synthetic experimentation. This, in turn, further accelerated the creation of new heuristic combinatory rules offering increasing control on chemical reaction paths at such a level that the concept of molecular design has now emerged as a realistic objective [23].

However, looking at the many ways in which chemical theories, all fundamentally inadequate and ill-defined at times (especially simple molecular orbital (MO) theory) not only explained things, but predicted new phenomena and new molecules, we understand that the strength of chemical theories is that chemistry makes large use of incommensurate theoretical frameworks of understanding and ill-defined concepts such as electronegativity, donor acceptor ideas, valence etc. These varied concepts, each with its own history, allow chemists to make sense and construct a world, when our theoretical knowledge is inadequate.

In other words, fuzziness and incommensurability are central to emergence, namely of moving from principles partially understood to real creation, to new properties. We recall here that in the philosophy of science, two theories are said to be incommensurable if there is no common theoretical language that can be used to compare them [24]. In other words, if two scientific theories are incommensurable, there is no way in which one can compare them to each other in order to determine which is better. Remarkably, such incommensurability "is taken by chemists without a blink, and actually serves" [25].

Let us take orbitals, for instance. Do they actually exist in real world? In 1999 direct observation of molecular orbitals (MO) was claimed (Figure 4), probed directly by photoelectron spectroscopy [26]. Immediately objection was raised that from a formal viewpoint there are no such objects as orbitals, and their "observation" is conceptually mistaken because quantum mechanics includes only the concept of wavefunction [27]. 


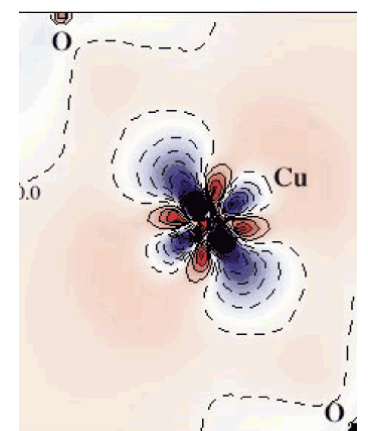

Figure 4. Molecular orbitals are mathematical functions in complex form Recently, their direct observation has been claimed (Reproduced from Ref [26], with permission)

This conceptual conflict on orbitals show how Feyerabend's argument that there are no single prescriptive methodological rules which are always used by scientists [28], applies equally well to chemistry (and not only to physics). Atomic orbitals are mathematical complex functions. In many-electron atoms, orbitals serve as a useful approximation to explain by the aufbau process many features of the periodic system [29], and serve as a basis set for the construction in molecular orbitals.

More generally, molecular orbitals, $\mathrm{sp}^{3}$ hybridization, $\pi$ bonds and related concepts are the results of the application of a specific computational method used to solve by approximation the Schrödinger equation for molecular systems. They are not experimentally based quantum laws that express, for instance, discreteness of certain physical quantities.

Orbitals however are also a (quantum) chemical model of immense importance in chemistry. Their relationship to the chemical methodology is heuristic, i.e., their usefulness in many branches of science justifies the use of the model. Now, chemists have used orbitals in every sense they want theory to be used -- to understand, to explain, to rationalize, to predict, to suggest syntheses. Those who reject orbitals based on a purist quantum mechanics approach, on the other hand, may well be formally correct; but they have done nothing for the making of new molecules, and thus of interest to chemistry.

For example, the reason organic chemists so quickly valued the work of Woodward and Hoffmann on the stereochemistry of pericyclic reactions based on orbital symmetry and molecular orbitals, is that it predicted in stereochemical detail the course of many organic reactions, and explained why others were difficult [30]. Similarly, by using early MO theory in 1943 Longuet-Higgins predicted the structure of diborane $\left(\mathrm{B}_{2} \mathrm{H}_{6}\right)$, an electron deficient molecule which could not be represented using normal covalent bonds; [31] and later he suggested the existence of the dodecaboride $\mathrm{B}_{2} \mathrm{H}_{12}$ dianion (while Pauling insisted on the neutral species); [32] as well as that of cyclobutadiene-iron-tricarbonyl, opening up two new fields of investigation. In addition, with the idea of through-bond coupling, and the perturbation theory underlying it, energy splittings and specific orderings of orbitals that were quite unexpected in azanaphthalenes were predicted; [33] and in 1960 the correct bent geometry of triplet methylene was computationally predicted [34].

\section{Chemistry as an autonomous discipline}

In 2000 Richard Bader commenting the historic development of the VSEPR (Valence Shell Electron Pair Repulsion) model of molecular geometry of Ron Gillespie emphasized that: [35]

"It seems difficult now to understand why, after the initial work by Lennard-Jones on the importance of the pair density to chemistry, its further investigation along other than purely formal lines was to languish for so long a period of time".
Boeyens indeed has recently shown at length how quantum ideas have often been inappropriately applied to chemistry, [1] suggesting that we should reconstitute a quantum theory appropriate to the conceptual demands of chemistry and one that focuses on the three-dimensional nature of molecules and their constituents.

Now, according to an attentive observer of chemical enterprise, today's chemists share a "variety of concerns suggestive of some underlying uncertainties and self-doubts" [36]. These "self-doubts" can be dispelled by reinforcing the idea that chemistry is an autonomous scientific discipline with its own logic and creative methodology. Such method originates a cornucopia of new, artificial substances which benefit society at large, and a better understanding of it has real benefits to chemistry practitioners and to chemical educators.

Practitioners of biology, mathematics, geology and physics all have a clear image of who they are and what their disciplines are intended for. However, a science widely perceived as socially "good" as biochemistry is naturally considered to be part of biology when actually is has been the interplay of chemistry and biology that originated this formidable discipline [37].

At the core of the chemical methodology there is a powerful approach based on mental visualization and association of chemical models for substances. These models can be molecular structures, a concept that originated from the solution of two chemical problems, namely those of valence and of optical isomerism. But they can also be synthetic "building blocks" of different size and shape, as the recent development of the chemical approach to nanomaterials -- nanochemistry [38]-- clearly shows. We know today that the modern picture of a molecule is not invariably equivalent to the classical molecular model of atoms joined by bonds, 4 and that the molecular structure is not an intrinsic property $[39,40]$.

But what is more relevant to the creative practice of a chemist: the synthesis of new functional substances, or the awareness that her/his model is "intrinsic"?

A scientific model is an abstract entity that selects from reality the variables that we consider relevant for our purposes. Our scientific theories do not refer directly to reality but to a model. A model is a particular kind of representation. And just like a representation of an object is not the object (Figure 5), a model of a system is not the system.

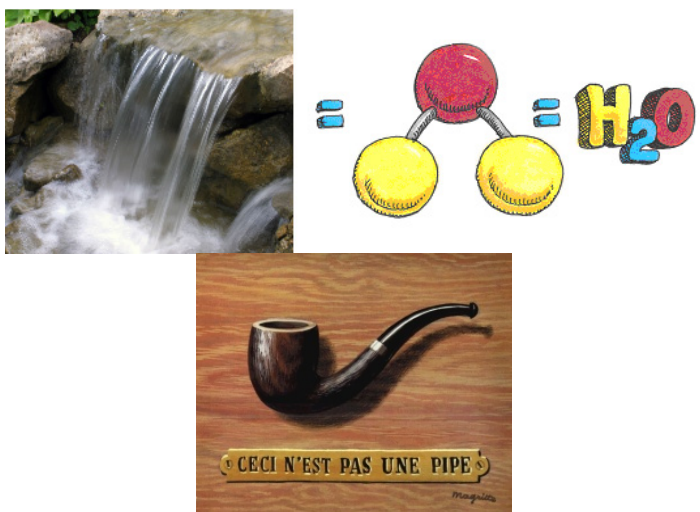

Figure 5. A representation is just a representation. Exactly like Magritte recalls us that the drawing of a pipe is not a pipe, a molecular structure is not a chemical substance.

We make hypotheses about the unobservable objects, properties and structure of the system under consideration and then we access real systems -- i.e., chemical substances -through our models. In other words, our direct access to real systems is invariably mediated by our ideas. This is what is meant by the idea of "representation" [41]. 
In chemistry too, the reductionist argument for there having to be one logical way to do things to be counted as adequate or successful is flawed [42]. Whereas chemistry accepts contributions of experimental findings, ideas, and models coming from other disciplines, chemistry has and keeps strong empirical and practical bases. Those deriving from quantum mechanics belong to this category. At this stage, it may be worth recalling Coulson's worries expressed in a famous after-dinner speech in 1959 [43].

Coulson was worried at that time that quantum chemistry, with the aid of the advent of electronic computers, could be reduced to a technique. To follow this way was in Coulson's opinion an error, leading to the development of a branch of computer mathematics of no relevance for the advancement of our understanding of the molecular world. The most important task of quantum chemical calculation was, according to Coulson, to provide more precise definitions of the "chemical concepts" originated in the chemical community, mostly on the basis of experience and of heuristic considerations. Actually Coulson's worries have been dispelled in the last decades, and theoretical chemistry has given substantial efforts to establish protocols of use and of defining limits of the tools of quantum chemistry, as well as to better define chemical concepts such as molecular structure and many others [44].

Eventually, continued improvements in implementation and reduction in the cost/performance of computing might lead us toward the "ultimate goal of theory" of achieving full partnership with experiment as both an explanatory and predictive methodology [45]. However, even in this wishful scenario, human chemical ingenuity that is central to the practice of the chemical methodology will not be replaced by computation.

\section{Pedagogical consequences and conclusion}

The concepts of chemical structure and chemical bonds emerged in chemistry independently and a long time ahead of quantum physics. Computational chemistry is not the best way to teach them for the simple reason that conceptual chemistry is not computational chemistry; the latter being a collection of approaches to solve by approximation the Schrödinger equation for those "complex atomic systems" identified by Dirac in 1929 - namely, chemical susbtances - for the equation simply cannot be solved exactly other than in the case of a oneelectron system (i.e., only for the hydrogen atom!). On the one hand, we chemists need a broader and better understanding of contemporary quantum mechanics. For instance, when electrons are treated as flexible standing waves, rather than point particles, such thinking enables us to deal with a large number of chemical concepts, including molecular structure, chemical bonding, chirality, reactivity and periodic classification of the elements [1].

On the other, we do need a better, more secure insight into chemical methodology as it evolved through the historic development of both chemical practice and theory. As brightly illustrated by Berson with numerous examples drawn from chemistry's enormously rich history, the synthesis of organic molecules has an "inescapably confirmative purpose". And this purpose comes from the modeling approach typical of the chemical ingenuity [46].

Did chemists fail to convey the beauty and magic of their science to the lay audience? Have we been able to show that chemistry has fundamental questions? With some notable exceptions, [47] the answer to the latter question is probably no.

Yet, we argue, these deficiencies are the result of a poor understanding among chemistry practitioners of the very fact that chemistry is a powerful, autonomous science with an intrinsic methodology that even upon the revolution of quantum mechanics, retains its great utility.
A few examples taken from the historical developments of chemical theory show that the strength of chemical theories lies in the plurality of theoretical models, often incommensurable, that constitute a rich conceptual body. This diversity needs not to be impoverished by a purist approach which has no influence on productive chemical research. Clearly, there is a danger in emphasizing the importance of the autonomous nature of a discipline, because fruitful progress is often made at the fuzzy interface between disciplines; and because great discoveries were often associated with the ability of the researcher to look at a problem from an angle which is outside her own discipline. Autonomy, however, is not synonymous of independence and collaboration with biologists, physicists, geologists etc. seeking the advice of chemists is and will increasingly be a feature common to leading chemical researchers.

\section{Acknowledgement}

This article is dedicated to the creative organic chemistry of University of Padua's Professor Sandro Campestrini. I thank Professor Roald Hoffmann (Cornell University, Ithaca) for his insightful comments to the original manuscript.

\section{References}

[1]. Boeyens, J. C. A. Chemistry From First Principles, Springer, 2008

[2]. Dirac, P. M. A. Proc. R. Soc. London, Ser. A 1929, 123, 714-733.

[3]. Mulliken, R. Phys. Rev. 1932, 41, 49-71.

[4]. Ampere, A. M. Ann. Chim. 1814, 90 (1), 43-86.

[5]. Atkins, P. W. Physical Chemistry, 4th edition, Oxford University Press, 1990.

[6]. Gillespie, R. J.; Humphreys, D. A.; Baird, N. C.; Robinson, E. A. Chemistry, Allyn and Bacon, 1986.

[7]. Suidan, L.; Badenhoop, J. K.; Glendening, E. J.; Weinhold, F. J. Chem. Ed. 1995, 72, 583-586.

[8]. Pimentel, G. C.; Spratley, R. D. Chemical Bonding Clarified Through Quantum Mechanics, Holden-Day, 1969.

[9]. Cotton, F. A.; Wilkinson, G. Advanced Inorganic Chemistry, John Wiley \& Sons, 1962

[10]. The late University of Palermo's Professor Giuseppe Werber.

[11]. Stone, D. C. Analyst 2005, 130, 419-420.

[12]. Schummer, J.; Bersnaude-Vincent, B.; Van Tiggelen, B. The Public Image of Chemistry, Imperial College Press, 2007.

[13]. Corey, E. J.; Cheng, X. M. The Logic of Chemical Synthesis, Wiley, 1995.

[14]. Geerlings, P.; De Proft, F. Int. J. Mol. Sci. 2002, 3, 276-309.

[15]. Ogilvie, J. F. J. Chem. Ed. 1990, 67, 280-289.

[16]. Jensen, F. Introduction to Computational Chemistry, John Wiley and Sons, 1999.

[17]. Bartlett, N. Proc. Chem. Soc. 1962, 27, 218-218.

[18]. Rundle, R. E. J. Phys. Chem. 1957, 61, 45-50.

[19]. Weisberg, R. Creativity, Beyond the Myth of Genius, W. H. Freeman, 1992.

[20]. Avnir, D.; Zabrodski Hel Or, H.; Mezey, P. G. J. Am. Chem. Soc. 1992, $114,7843-7851$

[21]. Alvarez, S.; Alemey, P.; Avnir, D. Chem. Soc. Rev. 2005, 34, 313-326.

[22]. Hoffmann, R.; Laszlo, P. Angew. Chem. Int. Ed. 1991, 30, 1-16.

[23]. Horváth, I. T. Acc. Chem. Res. 2002, 35, 685-694.

[24]. Dilworth, C. "Kuhn, Feyerabend, and Incommensurability", Chapter 7 , Scientific Progress. A Study Concerning the Nature of the Relation Between Successive Scientific Theories, pp. 49-54, Springer, 2007.

[25]. Hoffmann, R. Synthese 2007, 155, 321-336.

[26]. Zuo, J. M.; Kim, M.; O’Keefe, M.; Spence, J. C. H. Nature 1999, 401, 4952.

[27]. Wang, S. G.; Schwartz, W. H. E. Angew. Chem. Int. Ed. 2000, 39, $1757-$ 1762.

[28]. Feyerabend, P. K. Against Method: Outline of an Anarchistic Theory of Knowledge, Verso, 1975

[29]. Scerri, E. R. J. Chem. Ed. 2000, 77, 1492-1494.

[30]. Woodward, R. B.; Hoffmann, R. The Conservation of Orbital Symmetry, Verlag Chemie, 1970.

[31]. Longuet-Higgins, H. C.; Bell, R. P. J. Chem. Soc. 1943, 250-255.

[32]. Laszlo, P. Angew. Chem., Int. Ed. 2000, 39, 2071-2072.

[33]. Jordan, A. D.; Ross, I. G.; Hoffmann, R.; Swenson, J. R.; Gleiter, R. Chem. Phys. Lett. 1971, 10, 572-576.

[34]. Neugebauer, A.; Häfelinger, G. Int. J. Mol. Sci. 2005, 6, 157-176.

[35]. Bader, R. F. W. Coord. Chem. Rev. 2000, 197, 71-94.

[36]. Heylin, M. Chem. Eng. News 1998, 76 (2), 123-140.

[37]. Fruton, J. S. Proteins, Enzymes, Genes: The Interplay of Chemistry and Biology, Yale University Press, 1999. 
[38]. Ozin, G.; Arsenault, A. Nanochemistry: A Chemical Approach to Nanomaterials, RSC Publishing, 2005.

[39]. Wolley, R. G. J. Am. Chem. Soc. 1978, 100(4), 1073-1078.

[40]. Primas, H. Chemistry, Quantum Mechanics, and Reductionism, Springer, 1981.

[41]. Hesse, M. Models and Analogies in Science, University of Notre Dame Press, 1966

[42]. Lombardi, O.; Labarca, M. Found. Chem. 2005, 7, 125-148.

[43]. Coulson, C. A. Rev. Mod. Phys. 1960, 32, 170-177.

[44]. Jo Nye, M. J. Comput. Chem. 2007, 28(1), 98-108.

[45]. Friesner, R. A. Proc. Natl. Acad. Sci. USA 2005, 102, 6648-6653.

[46]. Berson, J. A. Chemical Discovery and the Logicians' Program. A Problematic Pairing, Wiley-VCH, 2003

[47]. Professor Pierre Laszlo: www.pierrelaszlo.net. 